\title{
Ethical challenges while conducting tobacco research among adolescents at community level and ways to handle them
}

Aryal UR

Assistant Professor, Department of Community Medicine, Kathmandu Medical College, Sinamangal, Kathmandu

G lobally, cigarette and other tobacco products are the leading causes of economic losses, morbidity and premature deaths. It is estimated that eighty percentage of tobacco related deaths occur in lowand middle-income developing countries ${ }^{1}$. Majority of adult smokers initiate smoking during adolescence and become addicted to smoking ${ }^{2}$. Later, these adolescents experience major health problems like cardiovascular diseases, cancer etc. in their life ${ }^{2}$. These are the reasons that tobacco smoking is a public health problem in both low- and middle-income and high- income countries. Therefore tobacco researches are essential to combat smoking. Expert panels, policy makers and stakeholders need to show concern on tobacco research among adolescents to implement effective intervention programme at community level. The results of tobacco research can produce significant benefits for the community to prevent smoking initiation among adolescents. For example, research has found that perceived benefits of cigarette smoking have led to increased smoking susceptibility among adolescents. Thus, the intervention programmes should focus on the fact that there is no any benefit of smoking ${ }^{3}$. Though tobacco researches among adolescents have many benefits to the society, a lot of ethical challenges and dilemmas arose during the community based survey ${ }^{3}$. These ethical challenges are similar to those mentioned in review articles published by Moolchan ET etal and Santelli JS et $\mathrm{al}^{4,5}$. Those common six challenges are mentioned in succeeding paragraph.

Firstly, most of the tobacco researches are based on basic principles of research i.e. autonomy of subjects (respect of the subjects), benefice and justice as described and explained in Belmont Report (1979) and Helsinki Declaration (1964) 4,5 . It is easy to explain these three principles in research proposal (or theoretically) but how to apply them in practice is a big challenge for the researchers. For example, when cross-sectional study, focus group discussion or any non-intervention study are conducted and it is found that many adolescents are smoking cigarettes, then it is duty of researchers to counsel the smokers "not to smoke" because smoking cigarettes causes cancer, heart diseases, and respiratory problemsetc.; createsfinancial burden to the person, their family and society; and also pollutes the environment from second-hand smoking. But many researchers fail to inform adverse effect to the participants as they think their objective is just to collect data and publish paper. This is unethical as the researcher fails to promote smoking cessation programme in the community. Thus, researchers should run intervention program to prevent smoking initiation and cessation in the community to protect health of the people or adolescents.

Secondly, when tobacco research is to be carried out among adolescents at community level who are below 18 years, the researchers are required to use both assent and consent form separately. It means that researchers are required to take permission at first from parents then from participants. His/her duty is to explain objective of the study very clearly otherwise there will be problem to get reliable data. Let us discuss it in Nepalese context where parents and guardians never believe that their children smoke cigarette unless they see it or hear it from the third person (either from their relatives or their neighbours). If the parents find their children smoking, they feel very bad and express their anger to their children. This is why smoker children are always sceptical about the enumerators that they might complain about their smoking habit to their parents. So they might hide their smoking activities. This is true when enumerators are hired locally. But there are also advantages of hiring local enumerators e.g. easy identification of the children in community and convenience to take permission from parents. Thus there is ethical dilemma for researchers whether to recruit local enumerators. In this situation, both local and other independent enumerators should 
be hired to get reliable data on smoking behaviours and it also helps to minimize the information bias. Some parents may be reluctant to give permission for participation in research believing that their children do not smoke. Again if the parents give permission to participate in the study then also smoker adolescents do not want to disclose their smoking habits. As a researcher, one has to respect the dignity of a person. So it is difficult for him/her to obtain data on smoking habits from face to face interview with each adolescent. Thus, self-administrated interview can be a good alternative where researcher can conveniently access adolescent smokers. The limitation of self-administrated interview is inconvenience for illiterate participants. Another hand sampling process may play vital role in the tobacco research to identify potential participants. When probability sampling technique is adopted, there is chance of getting all non-smoker participants. Therefore, respondent driven or snowball sampling will be appropriate to track smokers.

Thirdly, researchers and enumerators are mainly responsible to maintain confidentiality of data. When local enumerators are involved in data collection, there is a greater risk of revealing smoking status of adolescents to the community people and the parents, although they promise to keep confidentiality of data. While researchers want to implement intervention programme to those smokers after survey, he/she has to maintain confidentiality which is real ethical challenge for him/ her. In this situation, researchers have to inform smoking status of adolescents to the parents to participate in the program. To control the situation, researchers have to convince the parents in such way that there is no break up in child-parent relationship.

Fourthly, adolescents are voluntary participants in the tobacco research. Researchers should respect the time spent by participants but there is ethical dilemma whether to give remuneration for the study participants. There is high probability that the smoker can utilize remuneration for purchasing cigarettes. Instead of giving cash, it is better provide stationary items like diary, pen, pencils, copies, etc. so that both parents and participants are happy. It should be noted that the remuneration is not a benefit of participants in the study.

Fifthly, the ethical review board (ERB)plays an important role in tobacco research. ERB has the authority for modification or disapproval of research proposal, if proper guidelines are not followed. Evaluation of the research proposal depends upon the experience of reviewers and members of ERB on tobacco research. The reviewers should evaluate the proposal without prejudice, being flexible and with practical thinking. On the other side, there are limited numbers of tobacco researches in Nepal. Thus, its promotion is essential to control and prevent smoking among adolescents in Nepal.

Sixthly, local administrative authorities, health personnel and political leaders can also play significant role in tobacco research. Therefore, it is necessary to inform them objectives, methodology and benefits and harm to study participants to get permission from them. Otherwise, it is very difficult to get permission from the parents and to collect information from adolescents.

Finally, the researchers who are involved in tobacco research among adolescents should be familiar with ethical issues to improve quality of tobacco research. This will ultimately help to protect adolescents from the research risk.

\section{References}

1. World Health Organization. WHO report on the global tobacco epidemic, 2011: warning about the dangers of tobacco. Geneva: World Health Organization; 2011. use among teenagers: ethical challenges. J Adolescent Health. 2002;30(6):409-17.

2. US Department of Health and Human Services.A report of the Surgeon General: preventing tobacco use among young people.Washington DC: Department of Health and Human Services; 1994.

5. Santelli JS, Smith Rogers A, Rosenfeld WD, Durant $\mathrm{RH}$, Dubler $\mathrm{N}$, Morreale $\mathrm{M}$, et al. Guidelines for adolescent health research: A position paper of the Society for Adolescent Medicine. J Adolescent Health. 2003;33(5):396-409.

3. Aryal UR, Petzold M, Krettek A. Perceived risks and benefits of cigarette smoking among Nepalese adolescents: a population-based cross-sectional study. BMC Public Health. 2013;13(1):1-9.

4. Moolchan ET, Mermelstein R: Research on tobacco 